\title{
Using Normalised Sections for the Design of All Optical Networks
}

\author{
C. Caspar, R. Freund*, N. Hanik**, L. Molle*, C. Peucheret*** \\ Heinrich-Hertz-Institut für Nachrichtentechnik GmbH, Berlin; e-mail: caspar@hhi.de \\ *Virtual Photonics Incorporated, Berlin; e-mail: r.freund@virtualphotonics.com \\ **T-Nova, Technologiezentrum, Berlin; e-mail: norbert.hanik@telekom.de \\ *** Technical University of Denmark, Copenhagen; e-mail: cp@com.dtu.dk
}

Key words: optical networks, dispersion compensation, NRZ, numerical optimisation

\begin{abstract}
A novel concept for transparent link design is presented, and evaluated numerically and experimentally. $10 \mathrm{Gbit} / \mathrm{s}$ single channel transmission over more than $4000 \mathrm{~km}$ of Standard Single Mode Fibre is demonstrated. At reduced transmission distances, the systems show a high robustness against variations of the system parameters.
\end{abstract}

\section{INTRODUCTION}

Transparent optical WDM-systems are extremely increasing capacity and flexibility of today's information networks. The drawback of the network evolution towards transparent WDM is, however, that the almost perfect degree of network stability of periodically regenerated SDH-networks is lost. Concerning network planning, the complex interaction of several physical effects leads to specific limitations of the transmission distance to the socalled 'transparency-length'. In order to make wide use of the benefits of transparency, but also to avoid network instabilities, a future WDM-Network will consist of Transparent Optical Domains (TD) without 3R-regeneration, where a wide variety of data rates, data formats and wavelengths can be 
transported, and Subnetwork Interfaces (SNI) with 3R-regeneration for the interconnection of these Transparent Domains (Figure 1).

A strategy to simplify transparent network design and operation is to define so called 'Normalised Network Sections' which are cascaded to compose a particular transparent domain [1].

The optimisation of a specific Normalised Section is done in 2 steps: First, numerical simulations are performed for a single channel transmission system to identify the optimum parameter range and the system tolerances that can be expected. Due to this restriction, the calculation time is still within tolerable limits. In a second step, these numerical results are checked by experiments. Here, recirculating loop experiments were realised to imitate an arbitrary number of cascaded Normalised Transmission Sections. Finally, the performance of a WDM system using the so designed normalised section will show the limits for WDM transmission. Here, additional effects, e. g. Raman Scattering and Cross Phase Modulation [2], have to be taken into account. Nevertheless, the optimum range of operation parameters does not change significantly. In this paper, the optimisation of Single Channel Transmission is demonstrated.

The final result of this strategy is profound knowledge about the maximum extension of an optical network which applies the respective Normalised Transmission Section, and the parameter tolerances for networkoperation. The next section shows an example for this promising approach.

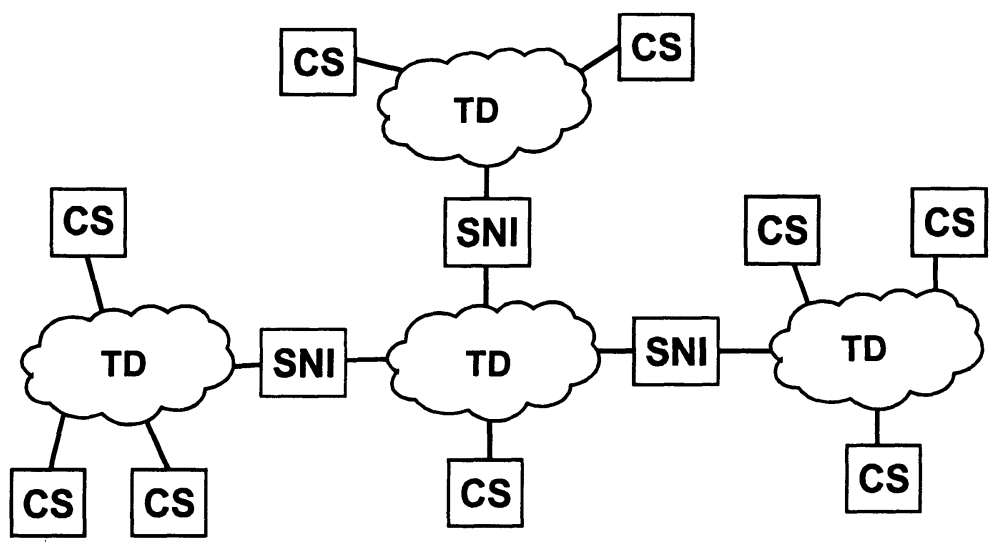

\section{TD: Transparent Domain \\ SNI: Subnetwork Interface \\ CS: Client Systems}

Figure 1. Vision of a future Optical Network 


\section{DESIGN AND OPTIMISATION OF THE TRANSPARENT DOMAIN}

\subsection{Specification of Normalised Transmission Sections}

As the design is performed for a maximum channel data rate of $10 \mathrm{Gbit} / \mathrm{s}$, the normalised sections have to be equipped with dispersion compensating modules. The arrangement, however, of deployed Standard Single Mode fibres (SMF, which are used in most of the European network), chromatic Dispersion Compensating Fibres (DCF) and Erbium Doped Fibre Amplifiers (EDFA) to compensate for the fibre attenuation, is variable, and may be a compromise between performance and costs.

Figure 2 shows two examples of normalised transmission sections based on SMF and Dispersion Compensating Fibre (DCF) using different schemes for the chromatic dispersion compensation. In both cases, the compensation ratio (CR) as well as the SMF/DCF input power can be varied independently. In case of pre-compensation, the additional (dotted) SMF in the transmitter section and the corresponding DCF in the receiver section allow changes of the so called "pre-distortion".

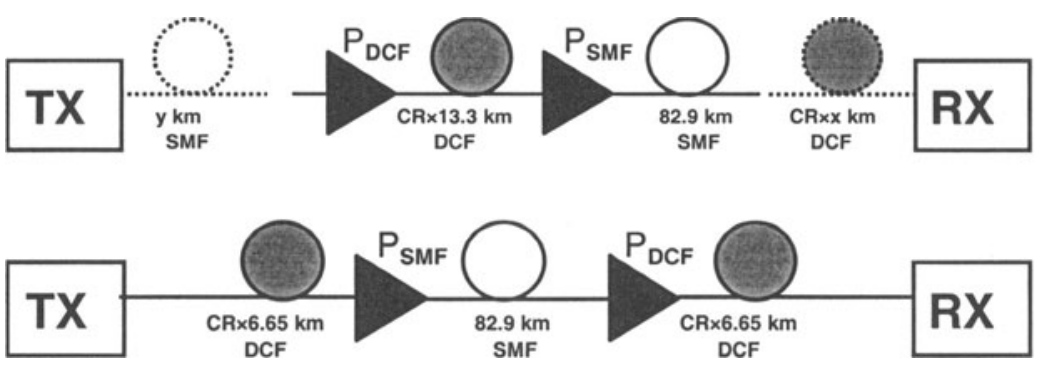

(a)

Figure 2. Normalised sections using pre-compensation (a) and hybrid-split (pre- and post-) compensation technique (b)

\subsection{Numerical investigation of the Normalised Section}

Objective of the optimisation process is to find the maximum number of cascadable sections with sufficient signal quality at the receiver. Therefore the power launched into the SMF, the power launched into the DCF, and the dispersion compensation ratio were independently varied. Table 1 gives an overview of the system parameters of the experimental setup (section 2.3) 
which were also used in the simulations. This numerical simulations were done by solving the Nonlinear Schroedinger Equation [3] applying the SplitStep-Fourier method. Due to the high SMF/DCF dispersion coefficient, the simulations are based on the assumption of independent signal and noise propagation. The Q-factor [4] was calculated under the assumption of Gaussian noise at the receiver, which gives a rather conservative estimation of the system performance.

Table 1. Parameters of the loop set-up (s. Figure 5), used in simulations.

\begin{tabular}{|l|c|c|}
\hline & SMF & DCF \\
\hline fibre attenuation $[\mathrm{dB} / \mathrm{km}]$ & 0.19 & 0.5 \\
\hline dispersion coefficient @ $1.55 \mu \mathrm{m}\left[\mathrm{ps} /\left(\mathrm{km}^{*} \mathrm{~nm}\right)\right]$ & 16.6 & -103.4 \\
\hline dispersion slope $@ 1.55 \mu \mathrm{m}\left[\mathrm{ps} /\left(\mathrm{km}^{*} \mathrm{~nm}^{2}\right)\right]$ & 0.06 & -0.207 \\
\hline non-linear refractive index $\left[\mathrm{m}^{2} / \mathrm{W}\right]$ & $2.6^{*} 10^{-20}$ & $2.6^{*} 10^{-20}$ \\
\hline effective core area $\left[\mu \mathrm{m}^{2}\right]$ & 69.5 & 19.0 \\
\hline maximum Raman gain $\left[\mathrm{m}^{2} / \mathrm{W}\right]$ & $3.2^{*} 10^{-14}$ & $3.2^{*} 10^{-14}$ \\
\hline EDFA noise figure: & $\approx 4.5 \mathrm{~dB}$ \\
\hline attenuation of DCF fibre splices: & $0.7 \mathrm{~dB}$ \\
\hline transmitter: & chirpless, external modulation \\
\hline optical receiver bandwidth: & $120.0 \mathrm{GHz}$ \\
\hline electrical receiver bandwidth: & $7.5 \mathrm{GHz}$ \\
\hline data signal: & $10 \mathrm{~Gb} / \mathrm{s}, \mathrm{NRZ}$ \\
\hline back-to-back receiver sensitivity $\left(\mathrm{BER}=10^{-9}\right):$ & $-37 \mathrm{dBm}$ \\
\hline
\end{tabular}

Figure 3 and Figure 4 show selected results of the numerical investigation. The power penalty shown in these figures is defined as the required increase of receiver input power to maintain a BER of $10^{-9}$ with respect to the back-to-back receiver sensitivity. The power levels into SMF and DCF, and the Compensation Ratio were varied. CR values between $98 \%$ (or $96 \%$ in case of the hybrid scheme) and $101 \%$ were evaluated. In Figure 3 , the best case $(\mathrm{CR}=99 \%$, Pre-distortion $=40 \mathrm{~km})$ and worst case $(\mathrm{CR}=101 \%$, Pre-distortion $=0 \mathrm{~km})$ for the normalised section with precompensation are shown. In the worst case, a transmission distance of 1658 $\mathrm{km}$ ( 20 cascades) results in a penalty of at least $4 \mathrm{~dB}$, whereas in best case, 40 normalised sections $(3316 \mathrm{~km})$ can be cascaded with a penalty less than 2 $\mathrm{dB}$. For the hybrid-split compensation scheme (Figure 4), a CR value of 99\% was identified as optimum. After 20 cascades a penalty less than $1 \mathrm{~dB}$ is obtained over a wide range of fibre input powers. After 40 cascades, a penalty of less than $3 \mathrm{~dB}$ is expected in the optimum parameter range. 

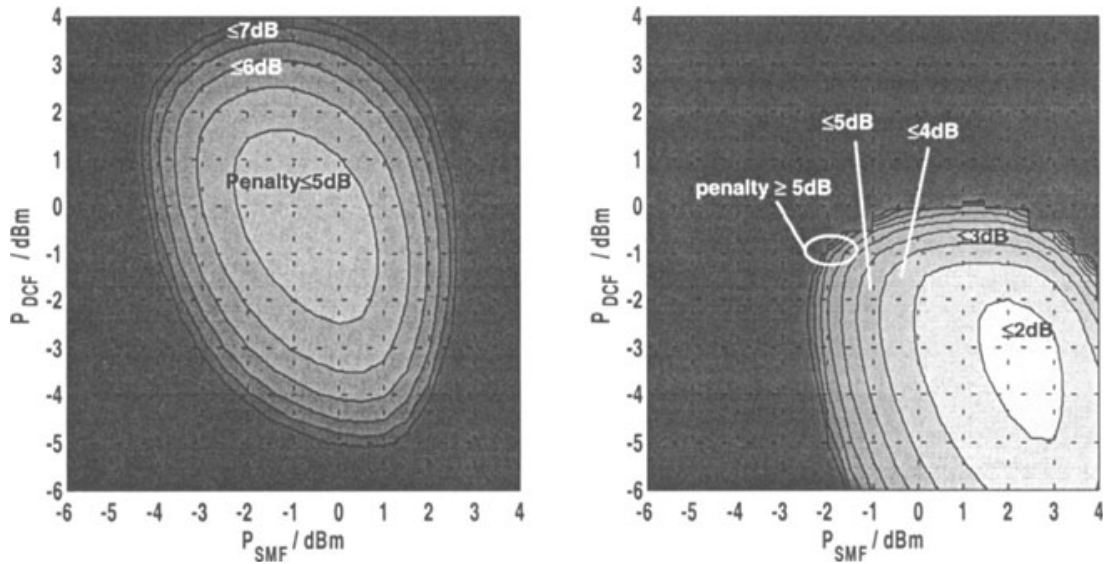

Figure 3. Simulated receiver sensitivity penalties for pre-compensation scheme: 20 loops, $\mathrm{CR}=101 \%$, Pre-Distortion $0.0 \mathrm{~km}$ (left, worst case); 40 loops, $\mathrm{CR}=99 \%$, PreDistortion $=40.0 \mathrm{~km}$ (right, best case).

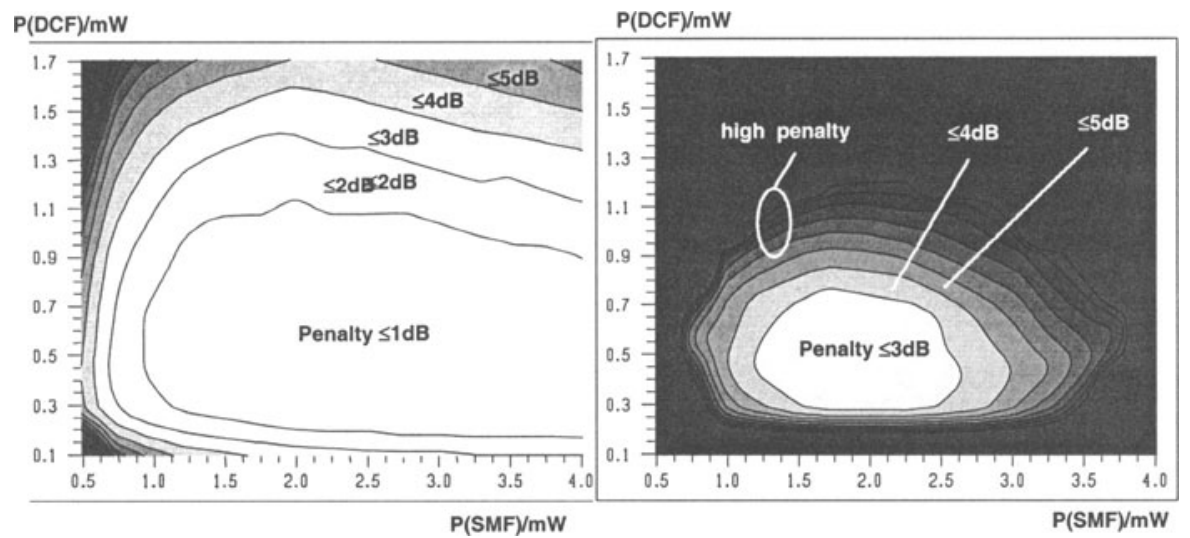

Figure 4. Numerical results for hybrid-split compensation scheme for $\mathrm{CR}=99 \%$ (best case) after 20 cascades (left) and 40 cascades (right). Penalty values of more than $5 \mathrm{~dB}$ are summarized by 'high penalty'. 


\subsection{Experimental validation of numerical simulations}

To verify the promising results of system simulations, fibre loop experiments (s. Figure 5, Table 1) were realised. To adjust the compensation ratio, small pieces of additional SMF were inserted in the loop, as well as the operating wavelength was slightly changed. An additional EDFA was used to compensate for the loop attenuation mainly given by the 3-dB coupler and the insertion loss of the loop switch. For the pre-compensation scheme, Figure 6 shows the penalty vs. the number of cascaded normalised sections for different compensation ratios and in the case of $40 \mathrm{~km}$ SMF predistortion. The penalties were measured for the respective optimum of the SMF/DCF input powers.

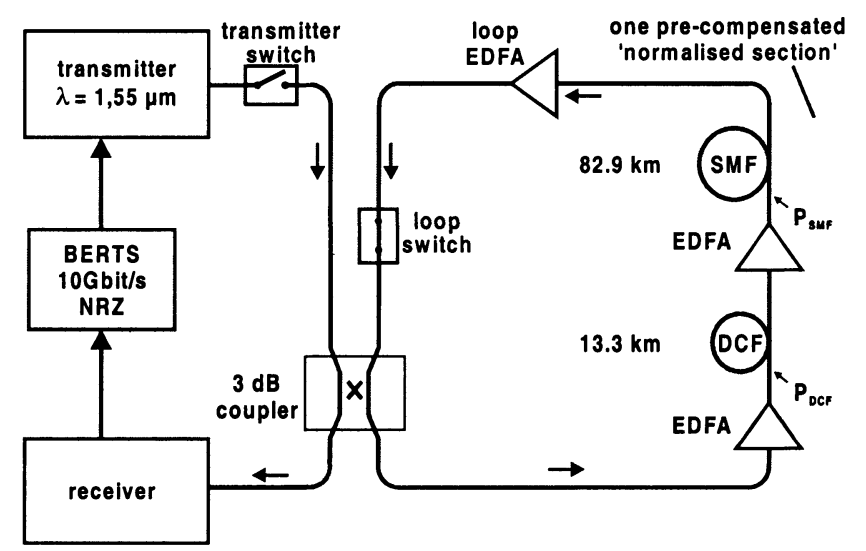

Figure 5. Fibre loop set-up for pre-compensated normalised section.

Without pre-distortion, Figure 6 shows an optimum system performance for a compensation ratio of $99 \%-100 \%$. In this range, 25 normalised sections $(2072,5 \mathrm{~km})$ can be cascaded with less than $4 \mathrm{~dB}$ penalty. It can be seen that due to additional pre-distortion a $10 \mathrm{~Gb} / \mathrm{s} \mathrm{NRZ} \mathrm{signal} \mathrm{can} \mathrm{be} \mathrm{transmitted}$ over more than $4000 \mathrm{~km}$. 


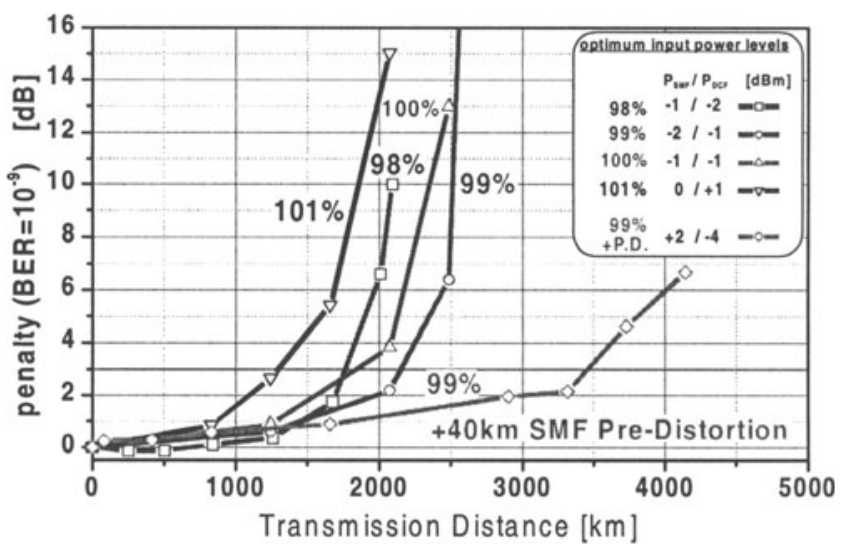

Figure 6. Ultimate transmission distances for the pre-compensation scheme for varying compensation ratio (measurements for $10 \mathrm{~Gb} / \mathrm{s}, \mathrm{NRZ}$ ).

Measurement (Figure 7) and simulations (Figure 3.) show very good concurrence. As it was predicted by simulations, in case of pre-distortion the optimum power region moves toward lower DCF and higher SMF input power. Because the values for the optimum input power strongly depend on the noise dependence of the individual amplifiers, the simulations shown in Figure 3 also take into account the measured gain saturation of the amplifiers, the measured input power dependence of the EDFA noise figures, and the additional losses due to the connectors in the transmission path.
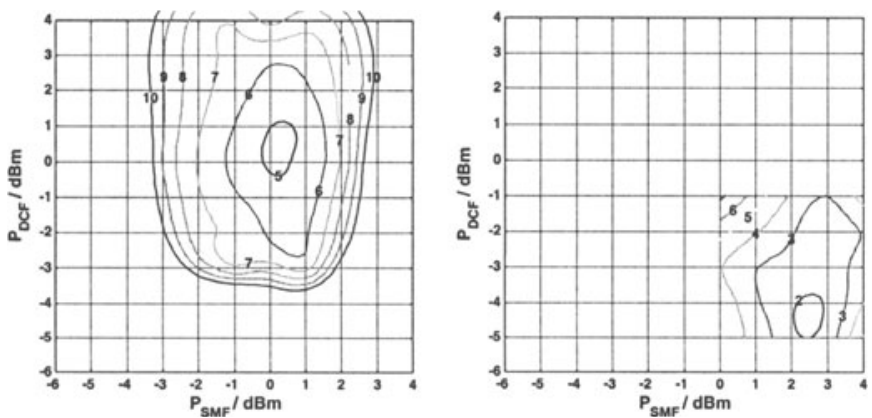

Figure 7. Measured receiver sensitivity penalties for pre-compensation scheme: 20 loops, $\mathrm{CR}=101 \%$, Pre-Distortion $0.0 \mathrm{~km}$ (left, worst case); 40 loops, $\mathrm{CR}=99 \%$, PreDistortion $=40.0 \mathrm{~km}$ (right, best case). 
Figure 8 depicts the experimental setup for the hybrid compensation scheme. In this case, 2 transmission sections are realised in the loop.

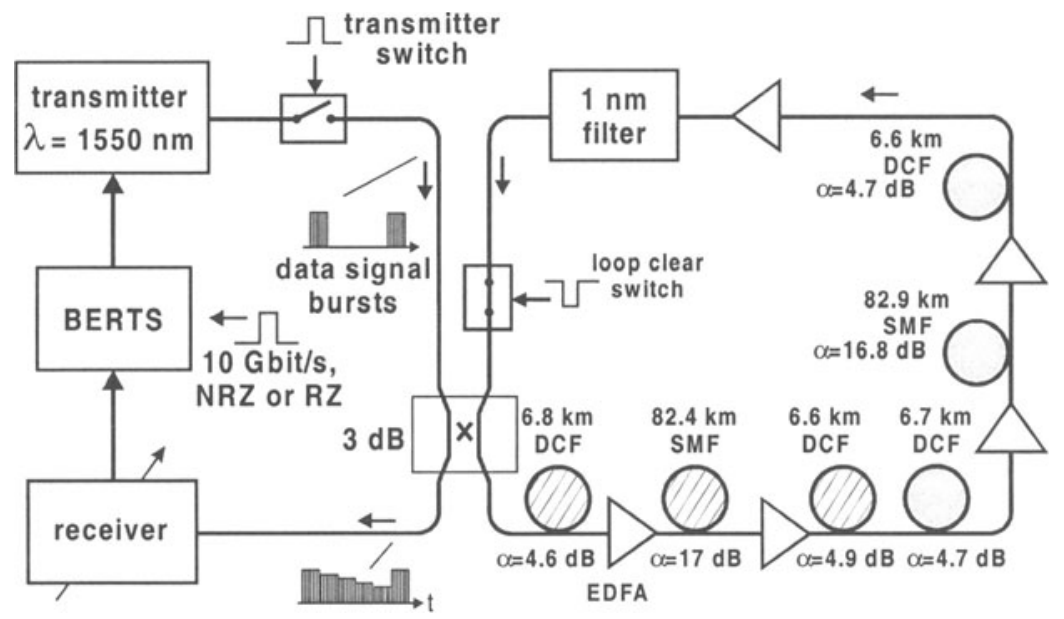

Figure 8. Experimental setup for the hybrid compensation method.

In Figure 9 the ultimate transmission distances using this hybrid-split compensation technique are shown for different degrees of compensation. The power levels are selected for maximum cascadability, respectively. In the optimum operating range of $99 \%$ compensation ratio, 50 cascades $(4145 \mathrm{~km})$ result in a penalty of less than $3 \mathrm{~dB}$. Simulations and measurements show very good concurrence.

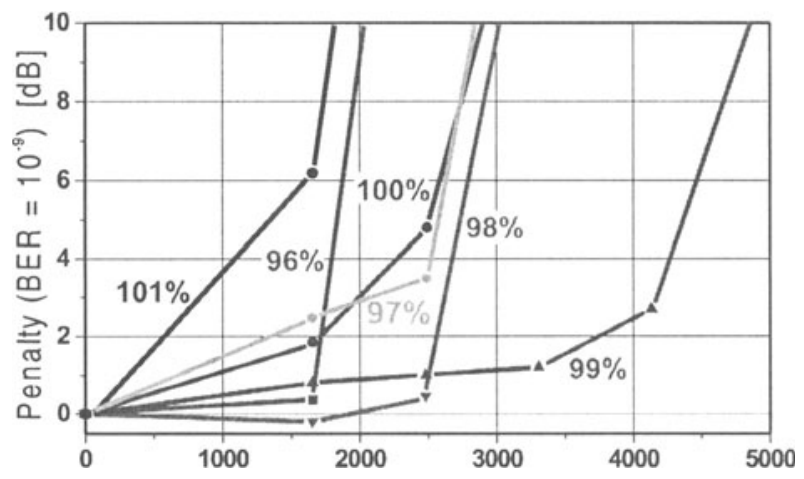

Figure 9. Ultimate transmission distances for the hybrid-split scheme for varying compensation ratio (measurements for $10 \mathrm{~Gb} / \mathrm{s}, \mathrm{NRZ}$ ). 


\section{CONCLUSION}

A high amount of system simulations have been performed to investigate the optimum design and operation of transparent, dispersion managed transmission links. Time consuming system experiments have been carried out to verify the results. Evaluation of the numerical and experimental data lead to the following conclusions:

- At moderate transmission distances (up to $1600 \mathrm{~km}$ ), high variations of input power levels into each fibre segment, and large deviations of the compensation ratio from the ideal value of $99 \%$ are tolerated.

- It is important to notice that the ultimate transmission limit of 50 cascades can only be obtained if Polarisation Mode Dispersion is very small along the whole transmission link. (The fibre material installed in the ring showed PMD values of $\leq 0.1 \mathrm{ps} / \mathrm{Nkm}$ ) The fibres for such a cascade should therefore be selected very carefully, otherwise the link distance would be limited by PMD.

- With optimised link design, a single channel, $10 \mathrm{Gbit} / \mathrm{s}$ NRZ data stream can be transmitted over $4000 \mathrm{~km}$ of Standard Single Mode fibre with amplifier distances of $80 \mathrm{~km}$. This assumes sufficiently low values for fibre attenuation and polarisation effects like Polarisation Mode Dispersion, and Polarisation dependent Gain or Loss

- The two Normalised Section designs investigated here have comparable tolerance ranges for the input powers into SMF and DCF, and likewise overlapping tolerance ranges for the degree of dispersion compensation. This means that these designs can be operated with identical parameters, a fact that greatly simplifies the operation of transparent links.

- The optimisation process for the 'pre-distortion' of the pure precompensated sections converges towards a 'symmetrical' dispersion map around a dispersion value of zero. In the case of pre-compensation, an addition of a $40 \mathrm{~km}$ fibre piece at the transmitter (plus the appropriate DCF piece at the receiver) leads to optimum system performance. The resulting dispersion map is very similar to the 'hybrid' section design, where the symmetrical dispersion map is perfectly realised

Acknowledgement: This work was supported by the European Community under ACTS-DEMON. 


\section{REFERENCES}

[1] E.-U. Scheuing, F.-C. Tischer: Photonic network design based on reference circuits. Conference on Optical Network Design and Modelling. Vienna, February 24-25, 1997.

[2] N. Hanik: Nonlinear Jitter of Optical WDM-Systems due to Cross-PhaseModulation. NOC'97, Antwerpen, Proc. 'Core and ATM Networks', pp. 178-181.

[3] G. P. Agrawal: Nonlinear Fiber Optics. Academic Press Inc, (1994).

[4] C. J. Anderson and J. A. Lyle, Technique for evaluating system performance using $\mathrm{Q}$ in numerical simulations exhibiting intersymbol interference, Electronics Letters, 1994, vol. 30, no. 1, pp. 71-72. 